\title{
An Exploratory Study to Identify the Risk Factors for Relapse in Patients with Alcohol Dependence following Abstinence in Selected Drug de Addiction and Rehabilitation Centers of New Delhi, India
}

\author{
Binu Thomas ${ }^{1}$, Bindu Shaiju ${ }^{2}$, Veena Sharma ${ }^{3}$ \\ ${ }^{1}$ Lecturer, VSPMs College of Nursing, Digdoh Hills, Hingna, Nagpur- 440019, India \\ ${ }^{2}$ Assistant Professor, Rufaida College of Nursing, Jamia Hamdard, New Delhi- 440062, India. \\ ${ }^{3}$ Associate Professor, Rufaida College of Nursing, Jamia Hamdard, New Delhi- 440062, India.
}

\begin{abstract}
This study aimed to identify the risk factors for relapse in patients with alcohol dependence following abstinence. The study objectives were to identify the risk factors and risk level for relapse in patients with alcohol dependence following abstinence, to find out the motivational factors for quitting alcohol and the relationship between the risk factors for relapse and selected demographic variables. An exploratory survey design included 60 patients attending four drug de-addiction and rehabilitation centres in Delhi, India, using purposive sampling technique. Data collected through structured interview schedule was analyzed using descriptive and inferential statistics. Results revealed that most of the samples were at moderate risk for relapse. Significant relationship was found between the risk factors for relapse and age of the study subjects, with most of the study subjects in age group of 29-39 years falling in the high risk category for relapse. Testing personal control over alcohol dependence was the most risky factor which led to relapse. Personal factors like wanting to be a responsible person or to feel better physically etc. motivated the client most to quit alcohol. The study concludes that most of the patients with alcohol dependence following abstinence are always at a risk for relapse.
\end{abstract}

Keywords: Relapse, Abstinence, Alcohol Dependence, Motivation to Quit, Risk Factors for Relapse

\section{Introduction}

Mercedes Mc Cambridge said alcohol is a very patient drug. It will wait for the alcoholic to pick it up one more time. Substance dependence is a major problem worldwide, India being no exception. Although short-term treatment of this condition is quite effective, preventing relapse often proves to be far more challenging. A majority of such patients relapse within an year of starting treatment, the first three months being the most vulnerable period [1]. However, most of the researches done are on alcohol dependence. Relapse has been examined far less frequently.

Alcoholism has long been accepted as one of the nation's most prevalent health problems yet it has often been neglected on a national basis. Alcoholism has emerged in our time as a unique and highly specific illness [2]. For many years, excessive alcohol consumption has been seen as an individual problem and it is only recently that a social concern over alcohol abuse has begun to develop. Alcoholism encounters no barriers of age, sex, race or political system. Whilst alcoholism has profound effects upon the alcoholices physical, emotional and social functioning, the effects are not limited to the alcoholic himself but extended to those around him as well. Relapse is a common and distressing aspect of alcohol addiction, mediated by several biological and psychosocial factors. It is commonly accepted that relapse rate among patients treated for alcoholism is extremely high [1].
The meaning of relapse has changed over years. Relapse was originally seen as a failure of the individual in recovery [2]. Dennis defined relapsed as a process to going back to the same unhealthy actions that would entice the reusing of substance or drugs [3]. Normally, individuals who are involved in the relapse process would show changing signs be it their attitude, thinking, emotions or actions. Relapse always occurs due to specific cause [2].

So, it is important to consider the reasons- why alcoholics relapse and the reasons which make them prone to develop alcoholism. The occurrence of relapse is so prevalent that some clinicians accept relapse as an inevitable part of the disease of alcoholism [1]. Over the recent years, relapse of alcoholic patients has been a topic of public concern. Due to the increasing rate of relapse, there is an urgent need for something to be done.

\section{Literature Survey}

Sau, Mukherjee, Manna and Sanyal tried to find out the pattern of substance use, relapse rate, its association with various socio-demographic factors and treatment related issues. An observational study with cross-sectional design during April 2009-March 2010 at a de addiction center was conducted among 284 consecutive clients admitted with relapse. They were detoxified earlier in the same center. Data were collected by interviewing clients with schedule and clinical examination. Polydrug abusers (59.1\%) were common. Only $31.3 \%$ of the relapse cases took regular follow up. Significantly higher relapse episodes were present with increasing age, Muslim religion, marriage, poor 


\section{International Journal of Science and Research (IJSR) \\ ISSN (Online): 2319-7064 \\ Index Copernicus Value (2013): 6.14 | Impact Factor (2014): 5.611}

literacy, current unemployment, living in nuclear rather than joint family, early age of initiation, longer duration of abuse and no follow up [4].

Leach and Kranzler reviewed the literature on interpersonal stress and rejection sensitivity and examine how these factors increase the risk of relapse in individuals with alcohol or drug dependence. They began by considering the constructs of social pain and social threat, examining their evolutionary origins and their neuro anatomical, neuropsychological and neuro physiological dimensions. Review of the empirical evidence showed that intrapersonal traits and interpersonal environments interact to increase an addict's risk of relapse. They concluded that substance-dependent individuals with high trait rejection sensitivity and a critical interpersonal environment are particularly vulnerable to relapse to substance use [5]

Sharma, Upadhyaya, Bansal, Nijhawan and Sharma studied factors affecting relapse in substance abuse. A cross sectional study was conducted on 40 patients of substance abuse relapse and 40 abstinent subjects as control. All the subjects were assessed on socio-demographic characteristics and history of substance abuse, and then subjected to Family Typology Scale, Family Interaction Scale, Presumptive Stressful Life Events Scale, Eyesenckes Personality Questionnaire and Multidimensional Scale of Perceived Social Support. It was found that relapse was more in substance abusers of less than 30 years of age, those having lower educational and socio-economic status, unemployed, having family history of substance abuse and past history of crime [6]

Matoo, et al conducted a study on psychosocial factors associated with relapse in men with alcohol and opiod dependence. They reported that patients who relapsed were significantly more likely (i) to have a positive history of substance use and higher number of previous relapses; (ii) to be using maladaptive coping strategies; (iii) to have been exposed to a higher number of high risk situations; and (iv) have experienced a higher number of undesirable life events. Those who have remained abstinent tended to use significantly more number of coping strategies, principally adaptive once [1].

Witkiewitz, et al did a study on drinking trajectories following an initial lapse. They evaluated the time to first lapse and the trajectories of post lapse drinking in a sample of 563 individuals who received community alcohol treatment. Results showed a decreasing risk of lapsing over time. Analysis of demographics, distal risk factors, time to first lapse and coping behaviors as predictors of time to lapse and post lapse drinking trajectories indicated alcohol dependence and coping behavior were the strongest predictors of lapsing and post lapse drinking behavior [8]

\section{Materials and Methods}

A quantitative approach with exploratory survey design was used. In this study the conceptual framework was based on RP Model given by Marlatt and Gordon (1984) [9]. The study was conducted in various de addiction centres of New Delhi, namely; Vishvasniya De addiction centre, Govindpuri,
All Saints Cancer Trust, Sangam Vihar, Generation Care Foundation, Kalkaji, Sambandh Samiti, Tughlaqabad and Jeevan Raksha Foundation, Kalkaji with 60 samples who were selected through purposive, non probability sampling technique. Criteria for selection of samples was - Patients having at least one episode of relapse after abstinence from alcohol dependence and patients having relapse after abstinence from alcohol only. The patients with other substance use disorders along with alcohol dependence, patients admitted in the drug de addiction and rehabilitation centre for the very first time and patients having serious withdrawal symptoms were excluded from the study. Administrative permission was taken from the Institutional Review Board (I.R.B) of Jamia Hamdard, Delhi, to conduct the research study. A written informed consent was taken from each study subject in addition, coding of the participantse $^{\text {ee }}$ names was done which ensured their anonymity.

A structured interview schedule to assess the risk factors for relapse in patients with alcohol dependence after abstinence and motivational factors for quitting alcohol was used for data collection. It was divided in 3 parts- Part A consisted of Demographic data, Part B consisted of a structured interview schedule to assess the risk factors for relapse in patients with alcohol dependence after abstinence. It had 102 Items. This part of the tool was based on the work of Marlatt \& Gordon who studied the concept of abstinence and relapse. Part $\mathrm{C}$ was a structured interview schedule to assess motivational factors for quitting alcohol with total of 58 items to find out the motivational factors for quitting alcohol. Reliability of the structured interview schedule was established by Cronbach's Alpha and the reliability coefficient was found to be 0.92 . The tool was of sufficient reliability for the purpose of the study. After taking formal administrative permission for conducting the final study, final data was collected. The data collected from the 60 study subjects was planned for analysis using both descriptive and inferential statistics.

\section{Results/Discussion}

Most of sample subjects, that is, $41(68.33 \%)$ were at moderate risk for relapse. Testing personal control was the most risky factor which led to relapse in patients with alcohol dependence. Testing one's personal control was identified as high risk situation to precipitate relapse in the study which was in accordance with Marlette s model [9]. Physical discomfort, which is a personal state was the least risky situation which led to relapse in patients with alcohol dependence following abstinence. Miller and Hester concluded in their study that about $50 \%$ of detoxified alcoholics relapse within three months [10]. In the present study, the duration of most recent abstinence episode was less than three months for most of the study subjects. The commonest motivational factors for quitting alcohol were in the areas of „Personal Factors ${ }^{\text {ee }}$ followed by ,Financial ${ }^{\text {le }}$ and „Social Factors". The obtained Fisher Exact"s value to establish relationship between the risk factors for relapse and age of the study subjects was found to be statistically significant at 0.05 level of significance, indicating that there was a significant relationship between age and risk for relapse. Although none of the study subject in the present study was below 18 years of age, but, studies from around 


\section{International Journal of Science and Research (IJSR) \\ ISSN (Online): 2319-7064}

Index Copernicus Value (2013): 6.14 | Impact Factor (2014): 5.611

the globe reveal the usual age of onset of alcohol used to be around 15 years with earlier onset associated with higher dependence rates [11] Heavy drinking occurs usually between 18 and 22 years of age with dependence occurring in the early to mid-20s [11]-[12].

\section{Conclusion}

The study concludes that relapse is a common aspect of alcohol dependence and people treated for alcohol dependence are always at a risk for relapse. Various personal factors can motivate a client for quitting alcohol but testing personal control can lead a client to relapse.

\section{Future Scope}

- A similar study may be replicated on a larger sample covering the entire population of a region or part of a country.

- A similar study may be conducted for assessing the knowledge on risk factors for relapse to alcohol dependence in schools and colleges.

- A comparative study can be done to ascertain high risk factors for relapse to alcohol dependence and other drugs.

\section{References}

[1] Mattoo, S. K.; Chakrabarti, S.; Anjaiah, M., “ Psychosocial factors associated with relapse in men with alcohol or opioid dependence", Indian Journal of Medical Research;Dec2009, 130(6).

[2] Majeke Sarah, "The influence of social factors on the relapse of alcoholic patients", UJ Library and Information Center. 2002.

[3] Ibrahim F, Kumar N, "The influence of community on relapse addiction to drug use: Evidence from Malaysia", European Journal of Social Sciences. 2009; 11(3).

[4] Dennis, C.D., "Relapse Prevention: Treatment Alternatives and Counseling Aids". Tab Books Inc, Blue Ridge Summit, PA , 1986.

[5] Sau M, Mukherjee A, Manna N and Sanyal S., "Sociodemographic and substance use correlates of repeated relapse among patients presenting for relapse treatment at an addiction treatment center in Kolkata, India”, African Health Sciences. 2013 Sep; 13(3): 791799.

[6] Leach D, Kranzler HR, "An Interpersonal Model of Addiction Relapse. Addict Disord Their Treat", 2013 Dec;12(4):183-192.

[7] Sharma Amit, Upadhyaya S, Bansal P, Nijhawan M and Sharma, DK, "A study of factors affecting relapse in substance abuse. Indian Journal of Life Sciences", 2012; 2(1): 31-35.

[8] Witkiewitz K, Masyn KE., "Drinking trajectories following an initial lapse. Psychology of Addictive Behaviors", 2008;22:157-167.

[9] Marlatt, A.G, George W.H, "Relapse Prevention: Introduction and Overview of the Modele, British Journal of Addiction 79 (1984), 261-273.

[10] Miller, W.R. and Heather, N., "The effectiveness of alcoholism treatment methods: what research reveals. In
Treating Addictive Behaviors: Process of Change", Plenum Press, New York. pp. 121-174.

[11] Schuckit MA, "Drug and alcohol abuse: A clinical guide to diagnosis and treatment", Springer; 2006.

[12] Clark DB, "The natural history of adolescent alcohol use disorders. Addiction", 2004;99:5-22.[PubMed].

\section{Author Profile}

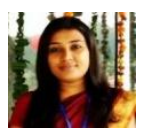

Ms.Binu Thomas received Master of Nursing degree from Rufaida College of Nursing, Jamia Hamdard, New Delhi, India. She has specialization in Mental Health and Psychiatric Nursing and is presently working as a Lecturer at VSPMs College of Nursing, Nagpur, Maharashtra, India. She is involved in teaching of under-graduate and post-graduate Nursing students, research guidance as well as organization and supervision of the clinical experience and other educational and administrative assignments.

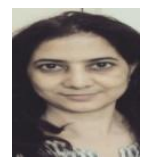

Ms. Veena Sharma received Master of Nursing degree from R.A.K. College of Nursing, Delhi University, India. She has specialization in Mental Health and Psychiatric Nursing., M.Phil. in Education from Central Institute of Nursing, Delhi University, India. She is presently working as Associate Professor at Faculty of Nursing, Jamia Hamdard, New Delhi, India. She is involved in teaching of undergraduate, post-graduate Nursing students, research guidance as well as organization and supervision of the clinical experience and other educational and administrative assignments.

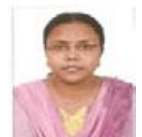

Ms. Bindu Shaiju obtained her Masters in Nursing (M.Sc. Psychiatry) from R.A.K. College of Nursing, New Delhi and B.Sc (Hons) in Nursing from All India Institute of Medical Sciences, New Delhi. She has to her credit over 20 yrs of experience in the areas of academic, clinical service in AIIMS, New Delhi and corporate arenas like Max Health Staff in NCLEX-RN training given for Indian nurses aspiring to work in U.S.A as well as ground research experience. Currently she is working as Assistant Professor at Jamia Hamdard in the Faculty of Nursing. 